THE ASTROPHySiCAL JouRNAL SUPPLEMENT SERIES, 90:561-565, 1994 February

(C) 1994. The American Astronomical Society. All rights reserved. Printed in U.S.A.

\title{
A THEORETICAL REVIEW OF DIFFUSIVE SHOCK ACCELERATION ${ }^{1}$
}

\author{
FRANK C. JONES \\ Laboratory for High-Energy Astrophysics, Code 665, NASA/Goddard Space Flight Center, Greenbelt, MD 20771 \\ Received 1993 February 26; accepted 1993 July 9
}

\begin{abstract}
We discuss the fundamental ideas of particle acceleration in plasma shocks with emphasis on those features that are required to produce the "universal" power-law spectrum. We compare shock acceleration with the more familiar second-order or stochastic acceleration and see that they are not too different in many respects. We discuss the features of shock acceleration that make it appealing and some of its problems as well.
\end{abstract}

Subject headings: acceleration of particles - MHD — plasmas - shock waves

\section{INTRODUCTION}

The notion that collisionless plasma shocks can accelerate charged particles to high energies has been around for a decade or so and by now it is widely accepted that this process can account for the majority, if not all, of the energetic, nonthermal, particle distributions that we see in astrophysical settings. I would like to present some of the fundamental ideas of the theory of diffusive shock acceleration, show what there is about the theory that makes it so attractive, and, perhaps demythologize the subject by demonstrating that it is not all that different from an old friend, namely, stochastic or secondorder Fermi acceleration. I will, therefore, begin with a discussion of stochastic acceleration.

\section{STOCHASTIC ACCELERATION}

The spectrum of particles that is produced by a stochastic acceleration process is derived simply from the equation of continuity in momentum space

$$
\frac{\partial}{\partial p}[\dot{p} f(p)]+\frac{f(p)}{T}=Q \delta\left(p-p_{0}\right)
$$

where $f(p)$ is the distribution function of the particles in scalar momentum space, $T$ is the mean time that particles stay in the accelerator, and $\dot{p}$ is the average rate of change of momentum produced by the acceleration process. A more rigorous treatment of stochastic acceleration would include a second derivative in momentum describing diffusion along the momentum axis. However, it has been shown that the resulting spectrum is of the same form (Morrison 1961) as the one resulting from equation (1) and evolves on the same time scale (Davis 1954), so that, except for treating specific models, nothing significant is gained by including such a term. The rate of momentum gain in stochastic acceleration has been discussed from many viewpoints: Fermi's original derivation (Fermi 1949), the resonant interaction of particles with Alfúen waves (Kennel \& Englemann 1966; Hall \& Sturrock 1967) or from a Boltzmann equation approach (Gleeson \& Axford 1967; Jones 1990). In

\footnotetext{
'Invited talk presented at the IAU Colloquium No. 142, "Particle Acceleration Phenomena in Astrophysical Plasmas."
}

each case it turns out that the momentum gain rate is proportional to the particles momentum. We may therefore set $\dot{p}=$ $\alpha p$, where $\alpha$ depends on properties of the scattering medium. This equation has the solution

$$
f(p)=\frac{Q}{\alpha p_{0}}\left(\frac{p}{p_{0}}\right)^{-(1+1 / \alpha T)}
$$

and is a power law in momentum provided the product $\alpha T$ is independent of momentum.

The problem with this approach is the independence of $\alpha$ and $T$; the power-law spectra that we see in nature tell us that the product $\alpha T$ must be of the order unity but nothing in most derivations requires this. However, it should be pointed out that recent work (Miller \& Ramaty 1992) has connected the two parameters in models in which plasma turbulence controls both the momentum gain rate and the rate with which particles diffuse out of the system.

\section{SHOCK ACCELERATION}

\subsection{Basic Ideas}

We begin by noting that, as in stochastic acceleration, shock acceleration theory shows that on each traversal of a shock a particle that is diffusing through the plasma gains an increment of momentum that is proportional to its momentum. Therefore after $N$ cycles through the shock a particle will have a momentum given by

$$
p(N)=p_{0} \prod_{i=1}^{N}\left(1+\left\langle\frac{\delta p}{p}\right\rangle_{i}\right)
$$

where the term in angle brackets is the flux-averaged, relative momentum increment on the $i$ th cycle through the shock. We label this term with the subscript $i$ because it may vary slowly as the particle continues to cycle through the shock. We shall consider a cycle to comprise a shock crossing from downstream to upstream, a second crossing from upstream to downstream and finally the subsequent return to the shock, a cycle, therefore, includes two actual crossings of the shock.

During each cycle there is a (assumed small) probability, $\epsilon_{i}$ that the particle will be swept downstream from the shock and never return. Thus the probability that the particle will actu- 
ally undergo $N$ cycles through the shock is given by

$$
\mathscr{P}(N)=\prod_{i=1}^{N}\left(1-\epsilon_{i}\right) .
$$

If we now take the logarithms of equations (3) and (4) we obtain

$$
\begin{aligned}
\ln \left[\frac{p(N)}{p_{0}}\right] & =\sum_{i=1}^{N} \ln \left(1+\left\langle\frac{\delta p}{p}\right\rangle_{i}\right) \\
& \approx \sum_{i=1}^{N}\left\langle\frac{\delta p}{p}\right\rangle_{i}, \\
\ln [\mathscr{P}(N)] & =\sum_{i=1}^{N} \ln \left(1-\epsilon_{i}\right) \\
& \approx-\sum_{i=1}^{N} \epsilon_{i},
\end{aligned}
$$

and then taking their ratio gives

$$
\frac{\ln \cdot \mathscr{P}(N)}{\ln \left[p(N) / p_{0}\right]}=\frac{-\sum_{i=1}^{N} \epsilon_{i}}{\sum_{i=1}^{N}\left\langle\frac{\delta p}{p}\right\rangle_{i}} \equiv-\Gamma(N ?),
$$

which leads to

$$
\mathscr{P}(p)=\left(\frac{p}{p_{0}}\right)^{-\Gamma(N ?)}
$$

which is the integral spectrum (what is the probability that a particle has a momentum of $p$ or greater). We have placed the question mark after the $N$ because we do not yet know whether or not $\Gamma$ depends on the number of cycles and hence momentum of a particle. If it does, then equation ( 8 ) is clearly not a power-law spectrum. To find out we must look at the terms $\langle\delta p / p\rangle_{i}$ and $\epsilon_{i}$ in more detail.

\subsection{Similarity to Stochastic Acceleration}

First let us note the similarity of the shock acceleration process to that of stochastic acceleration. The terms $\langle\delta p / p\rangle_{i}$ and $\epsilon_{i}$ represent the incremental momentum gain and probability of loss for a particle per cycle of the shock acceleration process. If $\tau_{i}$ is the time for one cycle we have

$$
\begin{gathered}
\frac{\sum_{i=1}^{N}\left\langle\frac{\delta p}{p}\right\rangle_{i}}{\sum_{i=1}^{N} \tau_{i}}=\frac{1}{p} \frac{d p}{d t}=\alpha(N), \\
\frac{\sum_{i=1}^{N} \epsilon_{i}}{\sum_{i=1}^{N} \tau_{i}}=\frac{\text { Probability of Loss }}{\text { Time }}=\frac{1}{T(N)} .
\end{gathered}
$$

We therefore have

$$
\begin{aligned}
\Gamma(N) & =\frac{\sum_{i=1}^{N} \epsilon_{i}}{\sum_{i=1}^{N}\left\langle\frac{\delta p}{p}\right\rangle_{i}}=\frac{\sum_{i=1}^{N} \epsilon_{i} / \sum_{i=1}^{N} \tau_{i}}{\sum_{i=1}^{N}\left\langle\frac{\delta p}{p}\right\rangle_{i} / \sum_{i=1}^{N} \tau_{i}} \\
& =\frac{1}{\alpha(N) T(N)},
\end{aligned}
$$

and the differential spectrum is given by

$$
f(p) \propto\left(\frac{p}{p_{0}}\right)^{-[1+(1 / \alpha(N) T(N))]},
$$

just as in stochastic acceleration. The spectrum depends on the same physical parameters in the same way and, as before, if the product $\alpha(N) T(N)$ is independent of $N$ (and hence momentum) the spectrum is a power law.

\subsection{Shock Acceleration Details}

To determine whether or not $\alpha(N) T(N)$ is independent of $N$ we must look further at the momentum gain term, $\delta p / p$ and the probability of return. It has been shown that for a particle, bound to a flowing plasma by scattering (Parker 1963; Gleeson \& Axford 1967) or by electromagnetic forces (i.e., drifts) (Jones 1990) the rate of change of its momentum is given by

$$
\dot{p}=-\frac{p}{3} \nabla \cdot \boldsymbol{u}
$$

where $\boldsymbol{u}$ is the flow velocity. If we take the normal of an infinite plane shock to be along the $x$ axis the only variations are along $x$ so we may write this as

$$
\dot{p}=-\frac{p}{3} \frac{\partial u}{\partial x} .
$$

Integrating equation (14) from the upstream side (side 1) to the downstream side ( side 2 ) along a particles trajectory gives

$$
\begin{aligned}
\delta p & =\int_{\text {side } 1}^{\text {side } 2} \dot{p} d t \\
& =-\frac{1}{3} \int_{\text {side } 1}^{\text {side } 2} p\left(\frac{d u}{d x}\right) \frac{d x}{v_{x}} \\
& =\frac{1}{3} \frac{p}{v_{x}}\left(u_{1}-u_{2}\right),
\end{aligned}
$$

where $v_{x}$ is the $x$ component of the particle's velocity.

We now wish the average value of $1 / v_{x}$ for those particles that cross the shock so we must weight the average by the flux of particles across the shock. Flux averaging gives

$$
\left\langle\frac{1}{v_{x}}\right\rangle_{\text {fux }}=\frac{2}{v} \text { (assuming isotropy) , }
$$

and since one cycle equals two traversals of the shock we have

$$
\left\langle\frac{\delta p}{p}\right\rangle_{i}=\frac{4}{3} \frac{\left(u_{1}-u_{2}\right)}{v_{i}} .
$$

The probability that a particle that crosses the shock from left to right will eventually return and cross the shock from right to left is simply the ratio of right to left flux to left to right flux. For particles of a given speed $v$ this may be readily computed. Looking at Figure 1 we see that in the downstream fluid rest frame the shock is moving to the left with a speed $-u_{2}$ and all particles with velocity vectors in the shaded cone can catch 


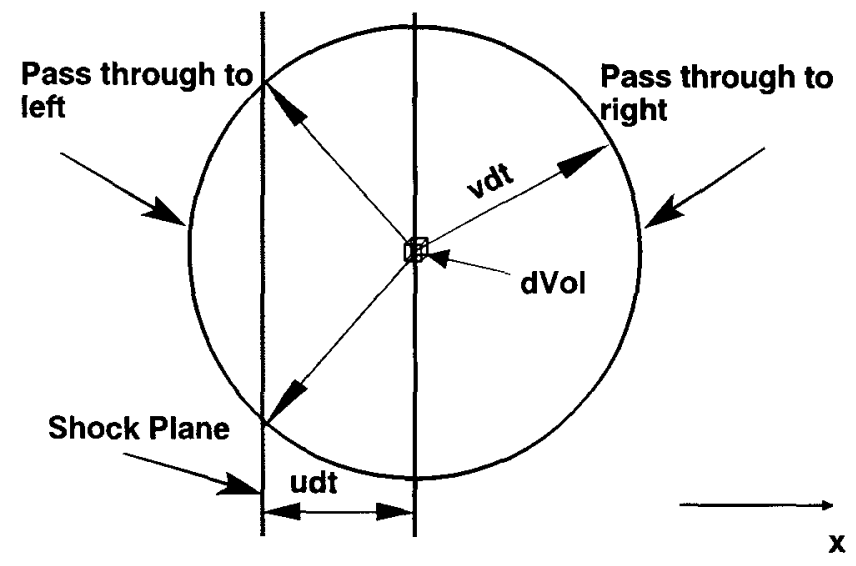

FiG. 1.-Geometry showing the relative flux of particles crossing a moving shock in either direction.

it and cross from left to right. Conversely, all other particles will cross the shock from right to left. The normalized fluxes of particles with speed $v$ are given by

$$
\frac{\left|\int_{-v}^{-u}\left(u+v_{x}\right) d v_{x}\right|}{\int_{-v}^{v} d v_{x}}=\frac{(v-u)^{2}}{4 v}
$$

for left to right and

$$
\frac{\left|\int_{-u}^{v}\left(u+v_{x}\right) d v_{x}\right|}{\int_{-v}^{v} d v_{x}}=\frac{(v+u)^{2}}{4 v}
$$

for right to left, all of which assumes that the particles are isotropic in the downstream fluid frame immediately behind the shock.

The probability of return is simply the ratio of the two above expressions and is given by

$$
p(i)=\frac{\left(v_{i}-u\right)^{2}}{\left(v_{i}+u\right)^{2}}=\left(\frac{1-u / v_{i}}{1+u / v_{i}}\right)^{2} \approx 1-\frac{4 u}{v_{i}}
$$

( for $u \ll v_{i}$ ).

In both of these derivations $v_{d}$ is the particles velocity on the $i$ th cycle through the shock.

If we now combine the above expressions, equations (17) and (20), to form the spectrum's "exponent" we have

$$
\begin{aligned}
\Gamma(N) & =\frac{4 u_{2} \sum_{i=1}^{N} \frac{1}{v_{i}}}{\frac{4}{3}\left(u_{1}-u_{2}\right) \sum_{i=1}^{N} \frac{1}{v_{i}}} \\
& =\frac{3 u_{2}}{u_{1}-u_{2}}=\frac{3}{r-1},
\end{aligned}
$$

where $r \equiv u_{1} / u_{2}$. We can see that, although the incremental momentum gain and probability of return were not themselves independent of $N$ and hence $p$, they were dependent in such a way that the dependence cancelled out in forming the exponent $\Gamma$. Furthermore, since for non relativistic, mon- atomic gases such as most astrophysical plasmas comprise, the compression ratio, $r$, is bounded from above by 4 so $\Gamma \geq 1$ and since many astrophysical shocks are strong (i.e., large Mach number) $r$ is close to 4 and thus $\Gamma$ is near 1 as is often observed.

Thus we see that the "magic" of the theory of shock acceleration is that the parameters $\alpha$ and $T$ are related in such a way that their product depends only on the compression ratio of the shock. Furthermore, astrophysical shocks tend to have compression ratios of the right order to produce observed spectra simply by being strong shocks.

\section{OBLIQUE SHOCKS}

\subsection{Problems with Geometry}

In the foregoing we have assumed that particles are free to move directly back and forth across the shock. This is true for a shock with no magnetic field or for a "parallel" shock, a shock in which the magnetic field is parallel to the shock normal. The more general case, however, is where the magnetic field makes an oblique angle, $\theta_{\mathrm{BN}}$ with the shock normal and inhibits the free motion of the particles across the shock. In this case the effective speed of the particle is reduced by the cosine of the obliquity angle and we have $v \rightarrow v \cos \Theta_{\mathrm{BN}}$.

We therefore have

$$
\begin{gathered}
\left\langle\frac{\delta p}{p}\right\rangle_{i}=\frac{4}{3} \frac{\left(u_{1}-u_{2}\right)}{v_{1} \cos \Theta_{\mathrm{BN}}}, \\
\mathscr{P}(i)=\frac{\left(v_{i}-u\right)^{2}}{\left(v_{i}+u\right)^{2}}=\left(\frac{1-u / v_{i}}{1+u / v_{i}}\right)^{2} \approx 1-\frac{4 u}{v_{i} \cos \Theta_{\mathrm{BN}}}
\end{gathered}
$$

so $\Gamma$ is unchanged provided $v_{\iota} \cos \Theta_{\mathrm{BN}} \gg u$. This condition will break down for some value of $\Theta_{\mathrm{BN}}$ no matter how large the particle speed $v$. In such a case, as in a perpendicular shock for instance, the only way that shock acceleration can proceed is for particles to be able to diffuse across the magnetic field. Even for moderate values of $\Theta_{\mathrm{BN}}$, however, there are always particles for which the above requirement is difficult, namely, those particles that are about to be injected into the shock acceleration process, the hot, shock-heated particles that have just traversed the shock for the first time. Computer simulations have shown (Jones \& Ellison 1991) that, for parallel shocks, these particles form a natural injector for the acceleration process, with sufficient numbers of them able to catch the shock from downstream and cross it to begin the climb to higher energies. The speeds of these particles, however, is only marginally larger than the downstream flow speed and a modest value of the obliquity angle can significantly reduce the number that can overtake the shock. For a view of the strong effect that a moderate value of $\Theta_{\mathrm{BN}}$ can have on the efficiency of shock acceleration in the absence of cross field diffusion, see the paper by Baring, Ellison, \& Jones ( 1994) in these proceedings.

\subsection{Cross Field Diffusion}

If shocks with a modest obliquity angle (and, of course, quasi-perpendicular shocks) are to be at all effective in accelerating particles there must be some process whereby particles can move across the magnetic field lines. It has been shown from different points of view (Forman, Jokipii, \& Owens 1974; 
Jones 1990) that whenever particles in a magnetic field are scattered in pitch angle to produce a diffusion coefficient parallel to the field given by

$$
\kappa_{\|}=\frac{1}{3} \frac{v^{2}}{\nu},
$$

where $\nu$ is the collision frequency, they also diffuse perpendicular to the field with a diffusion coefficient given by

$$
\kappa_{\perp}=\frac{1}{3} r_{g}^{2} \nu=\frac{1}{3} \frac{\left(r_{g} \nu\right)^{2}}{\nu},
$$

where $r_{g}$ is the particles gyroradius. The simplest interpretation of this result is that when a particle has its pitch angle scattered by a significant amount $(\approx \pi / 2)$ its guiding center is displaced perpendicular to the field in a random direction by an amount $\approx r_{g}$. We see from equation $(25)$ that the motion perpendicular and parallel to the field may be treated similarly provided we consider the quantity $r_{g} \nu$ to be the particles velocity perpendicular to the magnetic field.

If we add both components of $v_{x}$ to compute the mean value $\left\langle 1 / v_{x}\right\rangle_{\text {flux }}$ we obtain

$$
\left\langle\frac{\delta p}{p}\right\rangle_{i}=\frac{\left(u_{2}-u_{1}\right)}{\left(\frac{3}{4} v \cos \Theta_{\mathrm{BN}}+r_{g} \nu \sin \Theta_{\mathrm{BN}}\right)_{i}} .
$$

The probability of return is given by

$$
\begin{aligned}
& \mathscr{P}(i)=
\end{aligned}
$$

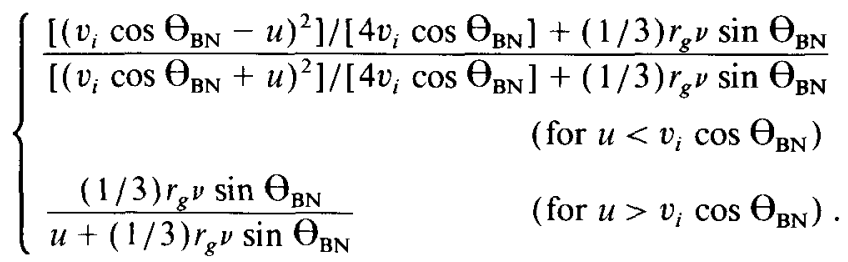

It should be noted that in equation (27) the flux due to cross field diffusion enters equally for the left to right flux and right to left flux, the flow velocity produces no asymmetry. This is because the guiding center is considered to jump instantaneously from one position to another so that the number jumping across a plane from any given direction is proportional to the particle density at that instant and is not affected by the flow speed.

Expanding equation (27) to first order in $u$ requiring that $u \ll v_{i}$ and that $u \ll r_{g} \nu$ we have

$$
\mathscr{P}(i) \approx \begin{cases}1-\frac{3 u}{r_{g} \nu \sin \theta_{\mathrm{BN}}+\frac{3}{4} v_{i} \cos \Theta_{\mathrm{BN}}} & \left(u<v_{i} \cos \theta_{\mathrm{BN}}\right) \\ 1-\frac{3 u}{r_{g} \nu \sin \theta_{\mathrm{BN}}} & \left(u>v_{i} \cos \theta_{\mathrm{BN}}\right)\end{cases}
$$

We should note, however, that the second form of equation (28) is really unnecessary, long before $v_{i} \cos \theta_{\mathrm{BN}}$ becomes less than $u$ it will have become negligible compared to $r_{g} \nu$ because for cross field diffusion to be effective at all in oblique or perpendicular shocks we were forced to require $r_{g} \nu \gg u$ and hence the first form of the equation may be taken to be generally valid.

If we now insert equations (28) and (26) into equation (7) we see once again that we obtain

$$
\Gamma=\frac{3 u_{2}}{u_{1}-u_{2}}=\frac{3}{r-1}
$$

and even though we do not yet know how much cross field diffusion will help the injection problem when $v \gtrsim u$ we can see that if the acceleration process does get started it will ultimately yield a power-law spectrum at high energies with the same power law as predicted for parallel shocks.

\section{TIMESCALES}

In closing, we will take one final look at the similarities between shock and stochastic acceleration. Stochastic acceleration is called "second order" because the relative momentum gain per collision is proportional to the second power of the (small) velocity ratio; i.e.,

$$
\left\langle\frac{\delta p}{p}\right\rangle \propto\left(\frac{u_{\mathrm{A}}}{v}\right)^{2}
$$

where $u_{\mathrm{A}}$ is the Alfvén speed, and the rate of momentum gain or acceleration rate is just this quantity times the collision frequency

$$
\left\langle\frac{1}{p} \frac{d p}{d t}\right\rangle \propto\left(\frac{v_{\mathrm{A}}}{v}\right)^{2} \nu
$$

Shock acceleration, on the other hand, is sometimes called "first-order Fermi" acceleration because the relative momentum gain per shock crossing is linear in the same ratio;

$$
\left\langle\frac{\delta p}{p}\right\rangle \propto \frac{\Delta u}{v} \approx \frac{u}{v},
$$

where $u$ is the plasma flow speed and $\Delta u$ is its change across the shock. As we have seen, the mean time a particle remains in the vicinity of the shock, $T$, is proportional to $\nu^{-1}(v / u)^{2}$ but the average number of times a particle crosses the shock during this period is given by

$$
\bar{N}=\sum_{N} N[\mathscr{P}(N)-\mathscr{P}(N+1)] \propto \frac{v}{u},
$$

so the crossing frequency (equivalent to the scattering frequency in stochastic acceleration ) is just ${ }^{2}$

$$
\frac{\bar{N}}{T}=\left(\frac{v}{u}\right)\left(\frac{u}{v}\right)^{2} \nu=\left(\frac{u}{v}\right) \nu,
$$


and thus the acceleration rate is

$$
\left\langle\frac{1}{p} \frac{d p}{d t}\right\rangle \propto\left(\frac{u}{v}\right)^{2} \nu
$$

which is just the same as for stochastic acceleration.

$$
\text { 6. CONCLUSION }
$$

We see, therefore, that shock acceleration has no particular advantage over stochastic acceleration in so far as speed is con- cerned. It is true that the speed $u$ in the shock acceleration rate is a (supersonic) flow speed which is usually faster than the Alfven speed $u_{\mathrm{A}}$ which appears in the stochastic acceleration but this is usually not a huge difference and can be quite insignificant when comparing different acceleration sites. The advantage that shock acceleration holds is its simple dependence of the spectral shape on the shock parameters and the fact that the particle spectra and the shocks that are observed in astrophysics seem to fit together quite well.
Baring, M., Ellison, D. C., \& Jones, F. C. 1994, ApJS, this issue Davis, L. 1954, Phys. Rev., 96, 743

Fermi, E. 1949, Phys. Rev., 75, 1169

Forman, M. A., Jokipii, J. R., \& Owens, A. J. 1974, ApJ, 192, 535

Gleeson, L. J., \& Axford, W. I. 1967, ApJ, 149, L1 15

Hall, D. E., \& Sturrock, P. A. 1967, Phys. Fluids, 10, 2620

Jones, F. C. 1990, ApJ, 361, 162

\section{REFERENCES}

Jones, F. C., \& Ellison, D. C. 1991, Space Sci. Rev., 58, 259

Kennel, C. F., \& Englemann, F. 1966, Phys. Fluids, 9, 2377

Miller, J. A., \& Ramaty, R. 1992, in Particle Acceleration in Cosmic Plasmas, ed. G. P. Zank \& T. K. Gaiser (New York: AIP), 223

Morrison, P. 1961, Handbuch der Physik, 46, 1

Parker, E. N. 1963, Interplanetary Dynamical Processes (New York: Wiley) 